\title{
Other and Unspecified Male Genital Organs ICD-O-3
}

National Cancer Institute

\section{Source}

National Cancer Institute. Other and Unspecified Male Genital Organs ICD-O-3. NCI

Thesaurus. Code C148341.

Male genital organs that are referenced in topographic category C63 of ICD-O-3. 